\title{
QCD in a finite box: Numerical test studies in the three Leutwyler-Smilga regimes
}

\author{
Stephan Dürr \\ Paul Scherrer Institut, Theory Group \\ 5232 Villigen PSI, Switzerland \\ stephan.duerr@psi.ch
}

\begin{abstract}
The Leutwyler-Smilga prediction regarding the (ir)relevance of the global topological charge for QCD in a finite box is subject to a test. To this end the lattice version of a suitably chosen analogue (massive 2-flavour Schwinger model) is analyzed in the small $(V \Sigma m \ll 1)$, intermediate $(V \Sigma m \simeq 1)$ and large $(V \Sigma m \gg 1)$ Leutwyler-Smilga regimes. The predictions for the small and large regimes are confirmed and illustrated. New results about the role of the functional determinant in all three regimes and about the sensitivity of physical observables on the topological charge in the intermediate regime are presented.
\end{abstract}

\section{Introduction}

One of the relevant concepts in an attempt to understand the mechanism of spontaneous breakdown of chiral symmetry" in QCD is provided by instantons, i.e. topologically nontrivial solutions of the classical field equations which are both a local minimum of the classical action and localized in spacetime. They are known to influence the local propagation properties of the light flavours (Instanton Liquid Model [1]).

What is not so clear is whether the global concept of the number of instantons minus anti-instantons for QCD in a finite box

$$
\nu=\frac{g^{2}}{32 \pi^{2}} \int G \tilde{G} d x
$$

plays a role, too. This is important, because in lattice studies involving dynamical fermions standard simulation algorithms tend to get "stuck" in a particular topological sector if the sea-quarks are taken sufficiently light? and obviously one would like to know whether this affects physical observables. In other words, the question is whether non-ergodicities of the sample w.r.t. the topological charge as visible e.g. from the r.h.s. of Fig. 1 tend to afflict measurements performed on such a sample.

\footnotetext{
${ }^{1}$ We shall only consider the theory with several light dynamical fermions $\left(N_{f} \geq 2\right)$.

${ }^{2}$ The phenomenon was first observed for HMC with staggered quarks [2]. For Wilson type sea-quarks mobility between the topological sectors was reported to be sufficient for $M_{\pi} / M_{\rho}>0.56$, but the same problem emerges if $\kappa$ is tuned sufficiently close to $\kappa_{\text {crit }}$, , 印.
} 

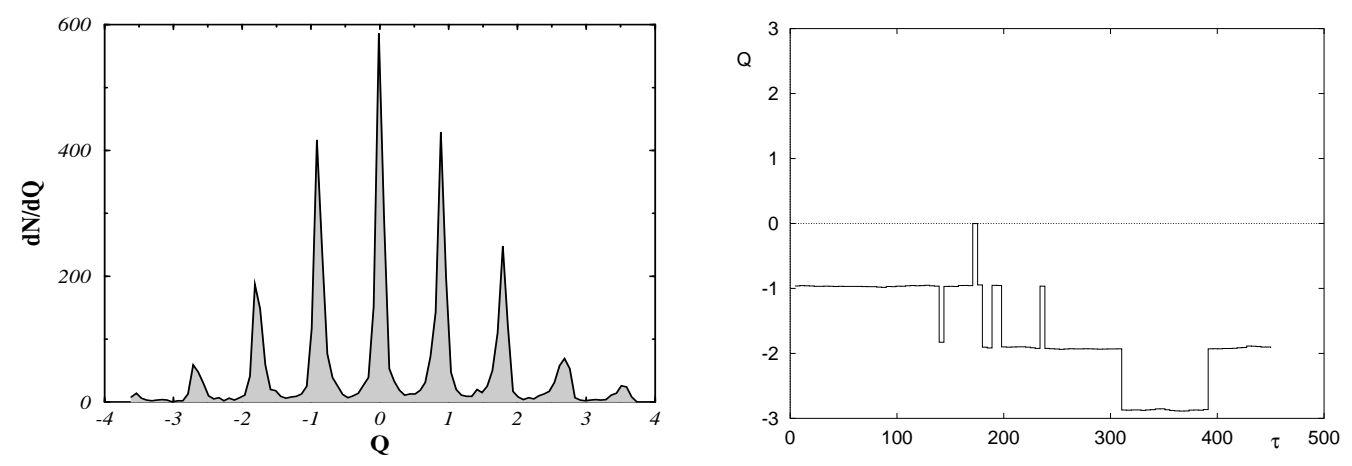

Figure 1: LHS: Distribution of the unrenormalized field-theoretic topological charge $\nu_{\text {nai }}$ in an ensemble of $5000 S U(3)$-configurations generated from $S_{\text {Wilson }}$ at $\beta=6.1$, after 6 cooling sweeps and with a 2-smeared charge operator (figure taken from [国]). RHS: Time history, in units of MD time, of $\nu_{\text {nai }}$ for a HMC simulation with a quadruple of staggered quarks at $\beta=5.35, m=0.01$; the analogous distribution lacks the symmetry $\nu \leftrightarrow-\nu$ and shows $\langle\nu\rangle \neq 0$ (figure taken from [ब]).

The correct sampling of the topological sectors is known to be crucial for quantities such as the $\eta^{\prime}$ mass, which depend directly on the distribution of topological charges via the explicit breaking of the $U(1)_{A}$ symmetry [5]. On the other hand, standard observables which do not relate to the $U(1)_{A}$ issue $\left(M_{\pi}, M_{K}, M_{\eta}, M_{\rho}, V_{q \bar{q}}, \ldots\right)$ are known not to depend on the global topological charge $\nu$ if the four-volume $V$ of the box is taken sufficiently large. An early investigation trying to assess the issue on a more quantitative level is the one by Leutwyler and Smilga [6]. Their main observation is that for the case of pionic observables a complete answer can be given from purely analytic considerations for two extreme regimes of quark masses and box volumes, both of which involve the Leutwyler-Smilga (LS) parameterf

$$
x \equiv V \Sigma m \text {. }
$$

Their first statement is that in a sufficiently small box $(x \ll 1)$ the question whether the algorithm managed to tunnel between the various topological sectors at a sufficient rate shouldn't emerge, because the partition function is completely dominated by the topologically trivial sector. In lattice language this amounts to the statement that the algorithm is not supposed to tunnel into a nontrivial sector anyways. Their second statement is that in the opposite regime $(x \gg 1$ plus a condition to be discussed below) the total topological charge is an irrelevant concept. This means, in lattice language, that the observable is unaffected by whichever "topological path" the simulation followed. From a lattice perspective, however, the analysis by Leutwyler and Smilga is not quite sufficient, as their paper doesn't state explicitly whether a simulation in the small $x$ regime can be trusted, if the algorithm got stuck in a higher topological sector even though it was not supposed to get into it, and also because there is no statement about the situation in the

\footnotetext{
${ }^{3} V$ is the four-volume of the box,$\Sigma=-\lim _{m \rightarrow 0} \lim _{V \rightarrow \infty}\langle\bar{\psi} \psi\rangle$ (this order) is the chiral condensate in the chiral limit and $m$ is the (degenerate) sea-quark mass; note that both $\Sigma$ and $m$ are scheme- and scale-dependent, but the combination is an RG-invariant quantity.
} 
intermediate $(x \simeq 1)$ regime, which might be interesting to use in dynamical simulations. Last but not least the relevance of the second condition in the LS-analysis for large $x$ (a condition which, as we shall see, is never fulfilled in QCD simulations) is not discussed.

Below numerical results will be used to elucidate in which regimes of box volumes and quark masses a standard observable like the heavy quark potential is indeed insensitive to the global topological charge and by which mechanism a dependence sneaks in if the "insensitive" regime is left. I start with a brief review of the approach taken by Leutwyler and Smilga, followed by an argument why the massive multi-flavour Schwinger model is a suitable testbed for numerical studies. Sections 4 through 6 present distinctive features by which the three LS-regimes differ from each other — both on a formal level (role being played by the functional determinant) and w.r.t. observables (Polyakov loop, heavy quark potential). The strategy used is to disentangle the complete sample into contributions from the individual topological sectors and to observe how the generating functional weights the fixed- $\nu$ expectation values to form the physical observable [7]. I conclude

with a few remarks on the potential relevance of the results for future QCD simulations.

\section{Leutwyler-Smilga analysis for QCD}

Here I shall briefly summarize the considerations by Leutwyler and Smilga regarding the distribution of topological charges for QCD in a finite box [6].

Leutwyler and Smilga start from the partition function for QCD with $N_{f} \geq 2$ degenerate light (dynamical) flavours on an euclidean torus

$$
Z \sim \sum_{\nu \in \mathbf{Z}} \int D A^{(\nu)} \operatorname{det}(\not D+m)^{N_{f}} e^{-(1 / 4) \int G G}
$$

where the integration is over all gauge potentials in a given topological sector and the quark fields have been integrated out. In (3) the Vafa Witten representation [8] for the functional determinant

$$
\operatorname{det}(\not D+m)^{N_{f}}=\left(m^{|\nu|} \prod^{\prime}\left(\lambda^{2}+m^{2}\right)\right)^{N_{f}}
$$

may be used which splits the latter into a factor representing the contribution from the zero modes of the Dirac operator $\not D$ on the given background times a chain of factors $\pm \mathrm{i} \lambda+m$ from the paired non-zero modes. Upon using the nontrivial input for the first few non-zero eigenvalues

$$
\lambda_{n} \simeq \frac{n \pi}{V \Sigma}
$$

(which is just the familiar Banks Casher relation [9]) the primed factor in the decomposition (4) is seen not to depend on $m$ if $x \ll 1$, and hence

$$
Z_{\nu} \sim m^{|\nu| N_{f}} \quad(x \ll 1) .
$$


This means that in the chiral symmetry restoration regime $(x \ll 1)$ topologically nontrivial sectors are heavily suppressed w.r.t. the trivial one - in particular for small quark masses (in lattice units) and large $N_{f}$.

On the other hand, if the box volume is sufficiently large, the onset of chiral symmetry breaking gets manifest as the long-range Green's functions get dominated by the Goldstone excitations and the theory is effectively described by a chiral lagrangianf

$$
Z \sim \int_{S U\left(N_{f}\right)} D U e^{-\int L_{\mathrm{XPT}}}, \quad L_{\mathrm{XPT}}=\frac{F^{2}}{4}\left\langle\partial U \partial U^{\dagger}\right\rangle-\frac{\Sigma}{2}\left\langle U M^{\dagger}+M U^{\dagger}\right\rangle
$$

where the integration is over all smooth fields taking values in $S U\left(N_{f}\right)$, and standard chiral perturbation theory (XPT) to order $O\left(p^{2}\right)$ has been used . In the representation (7) $U=\exp (\mathrm{i} \sqrt{2} \phi / F)$ is the Goldstone manifold and $M=\operatorname{diag}(m, \ldots, m)>0$ the quark mass matrix; $\langle\ldots\rangle$ means a flavour trace, and $F$ is the pion decay constant to $O\left(p^{2}\right)$. The key observation by Leutwyler and Smilga is that if they take not only the box length sufficiently large for XPT to apply' but also the quark masses sufficiently light

$$
\frac{1}{\Lambda_{\mathrm{XPT}}} \ll L \ll \frac{1}{M_{\pi}}
$$

the collective (constant mode) excitations are no longer suppressed, but provide the dominant contribution [11], i.e. (7) is approximately given by

$$
Z \sim \int d U_{0} e^{V \Sigma\left\langle U_{0} M^{\dagger}+M U_{0}^{\dagger}\right\rangle / 2}
$$

where the path integral has collapsed into a simple group integral given by the Haar measure. From (9) one gets the contribution of the topological sector $\nu$ to the partition function in the standard way by substituting $M \rightarrow M e^{\mathrm{i} \theta / N_{f}}$ and Fourier transforming from $\theta$ to $\nu$, whence

$$
\begin{aligned}
Z_{\nu} & \sim \int_{0}^{2 \pi} d \theta \int d U_{0} e^{-\mathrm{i} \nu \theta} e^{V \Sigma\left\langle U_{0} e^{-\mathrm{i} \theta / N_{f}} M^{\dagger}+\text { h.c. }\right\rangle / 2} \\
& \sim \int_{U\left(N_{f}\right)} d \tilde{U}(\operatorname{det} \tilde{U})^{\nu} e^{V \Sigma\left\langle\tilde{U} M^{\dagger}+\text { h.c. }\right\rangle / 2}
\end{aligned}
$$

where in the last line $U_{0}$ and the factor $e^{-\mathrm{i} \theta / N_{f}}$ have been combined into the $U\left(N_{f}\right)$ matrix $\tilde{U}$. From (10) it follows that, if $V \Sigma m$ is large, the dominant contribution to the group integral will come from the area where $\tilde{U}$ is in the vicinity of the identity matrix, which means that the determinant is approximatively one and hence $Z_{\nu}$ is independent of $\nu$. A refined analysis shows that this is true for $\nu^{2} \ll V \Sigma m / N_{f}$, and the overall distribution is [6]

$$
Z_{\nu} \sim e^{-\frac{\nu^{2}}{2\left\langle\nu^{2}\right\rangle}} \quad \text { with } \quad\left\langle\nu^{2}\right\rangle=\frac{V \Sigma m}{N_{f}} \quad(x \gg 1) .
$$

\footnotetext{
${ }^{4}$ References to all the subtle points (e.g. why in the particular case of the torus no boundary terms show up in $L_{\mathrm{XPT}}$ ) are found in [6].

${ }^{5}$ For a discussion in the framework of generalized chiral perturbation theory see 10 .

${ }^{6} \Lambda_{\mathrm{XPT}}$ denotes a QCD intrinsic low-energy scale, e.g. $\Lambda_{\mathrm{QCD}}, \sqrt{\sigma}, F_{\pi}$.
} 
I shall attach a few comments:

(i) It is worth noticing that the LS-classification of small, intermediate and large box volumes $(x \ll 1, x \simeq 1, x \gg 1)$ does not coincide with the usual classification on the lattice $\left(L \ll M_{\pi}^{-1}, L \simeq M_{\pi}^{-1}, L \gg M_{\pi}^{-1}\right)$. Note also that one involves the masses of the sea-quarks, the other of the current-quarks.

(ii) The analysis by Leutwyler and Smilga covers the symmetry restoration regime $(x \ll 1)$ and, on the other side, the regime where SSB is manifest, but the Goldstone bosons overlap the box $\left(x \gg 1, L \ll M_{\pi}^{-1}\right)$. The latter condition — besides not being useful because it prevents[] extraction of pionic observables - is, in fact, completely inaccessible in present days simulations: Putting numbers into eqn. (\$) one gets

$$
\Lambda_{\mathrm{XPT}} \simeq 200 \mathrm{MeV} \quad \Longrightarrow \quad L \simeq 3 \mathrm{fm} \quad \Longrightarrow \quad M_{\pi} \simeq 20 \mathrm{MeV}
$$

which amounts to a sea-quark mass of the order of $70 \mathrm{keV}$. From this we conclude that, in order to be useful in dynamical QCD simulations, the result (11) must turn out to rely exclusively on the condition $x \gg 1$ being fulfilled, not on the r.h.s. of (8). In other words, our hope is that the latter turns out to be a purely technical condition, immaterial to the result (11).

(iii) Comparing the announcement of the results by Leutwyler and Smilga in the introduction to their considerations as sketched above, one might worry because the latter aims at the relative weight of the topological sectors in the partition function, whereas the introduction mentioned possible $\nu$-dependencies of observables. From a formal point of view one may argue that this is not an issue, because the LS-analysis goes through if the theory is coupled to tiny external sources, and infinitesimal sources are sufficient to produce Green's functions and observables. Hence one expects that a strong dependence or approximate independence of the partition function w.r.t. $\nu$ transfers into a sensitivity or immunity of correlation functions and observables, but still one would desire to see this explicitly in numerical data.

\section{Adaptation to 2-flavour QED(2)}

The aim is to provide simulation data in the three LS-regimes $x \ll 1, x \simeq 1$ and $x \gg 1$, while in addition fulfilling the usual condition $L \gg 1 / M_{\pi}$, i.e. the opposite of the r.h.s. of (8).

A toy theory where the LS-issue can be studied at greatly reduced costs (in terms of CPU power) while maintaining all essential features is the massive multi-flavour Schwinger model, i.e. $\operatorname{QED}(2)$ with $N_{f} \geq 2$ light degenerate fermions. The physics of this theory resembles in many aspects QCD $(4)$ slightly above the chiral phase transition, i.e. the chiral condensate vanishes upon taking the chiral limit and the expectation value of the Polyakov loop is

\footnotetext{
${ }^{7}$ Unless the current-quark mass is taken considerably heavier than the sea-quark mass.

${ }^{8}$ Since $M_{\pi}^{2} \sim m$, a sea-pion which is lighter than the physical pion by a factor 7 amounts to a sea-quark mass 49 times smaller than the phenomenological value of $\left(m_{u}+m_{d}\right) / 2$. For quark masses the usual conventions $(\overline{\mathrm{MS}}, \mu=2 \mathrm{GeV})$ are adopted.
} 
real and positive. Furthermore, the required formal analogy holds true: Like in QCD the gauge fields in the continuum version of the Schwinger model quantized on the torus fall into topologically distinct classes [12], i.e. they can be attributed a topological index. The latter agrees with the number of left- minus right-handed zero-modes of the massless Dirac operator, i.e. the index theorem holds true 12.

There is, however, an (apparent) problem: As we have seen in the previous section, the physics in the large LS-regime $(x \gg 1)$ is governed by the onset of SSB for the global $S U\left(N_{f}\right)_{A}$ flavour-group (though the box volume is still finite), but it is well known that in 2 dimensions no SSB with the associate production of Goldstone bosons takes place [13].

The reason why this apparent deficiency proves immaterial relates to the fact that the multi-flavour Schwinger model (with $N_{f}$ massless flavours) shows a second order phase transition with critical temperature $T_{c}=0$, a result due to Smilga and Verbaarschot [14]. The theory is most appropriately seen as the limiting case of an extension where the axial flavour symmetry is explicitly broken, e.g. by tiny quark masses. In the strong-coupling limitf $m \ll g$ the spectrum is found to involve a heavy particle with mass [15]

$$
M_{+} \simeq \sqrt{N_{f}} \frac{g}{\sqrt{\pi}}+O(m)
$$

and $N_{f}^{2}-1$ light particles with mass [13, 16]

$$
M_{-} \simeq \text { const } g^{1 /\left(N_{f}+1\right)} m^{N_{f} /\left(N_{f}+1\right)} .
$$

The state (13) is often called a "massive photon", but it is more appropriately seen as the analogue of the $\eta^{\prime}$ in QCD, as it is the lightest flavour singlet and stays massive in the chiral limit. The states (14) are "Quasi-Goldstones" and we shall call them "pions", but it is important to keep in mind that they differ from the usual "Pseudo-Goldstones" (as encountered in QCD) as they do not turn into true Goldstone bosons upon taking $m \rightarrow 0$; they rather become sterile if the chiral limit is performed. That conforms with Coleman's theorem [13] which forbids the existence of massless interacting particles in 2 dimensions. The point I shall stress is this: As long as the axial flavour symmetry is explicitly broken — which, in the following, is true, as sea-quarks are taken massive — the "Quasi-Goldstones" are, for practical purposes, as good as "Pseudo-Goldstones"; the difference shows only up upon taking the chiral limit. Hence even the large LS-regime, where long-range Green's functions are dominated by pionic excitations, finds its analogue in the (massive) multi-flavour Schwinger model.

One more practical issue has to be resolved: The original definition (2) of the LS-parameter $x$ involves the quantity $\Sigma$, i.e. (the absolute value of)

\footnotetext{
${ }^{9}$ Note that in 2 dimensions the charge has the dimension of a mass.

${ }^{10}$ Analogous phenomena are observed, if the axial flavour symmetry is broken by boundary conditions rather than quark masses: The chiral condensate (i.e. the "would be order parameter") vanishes with a power-law dependence as the spatial box length is sent to infinity at zero temperature, but exponentially fast if the temperature is non-zero [17.
} 
the chiral condensate in the chiral limit. The latter, however, is exactly zero, due to Coleman's theorem. A practical way out of this is based on Smilga's observation that upon combining (14) with the expression [16]

$$
|\langle\bar{\psi} \psi\rangle| \simeq \text { const } g^{2 /\left(N_{f}+1\right)} m^{\left(N_{f}-1\right) /\left(N_{f}+1\right)}
$$

for the chiral condensate one gets the relation [16]

$$
m|\langle\bar{\psi} \psi\rangle| \simeq \frac{0.388}{(2.008)^{2}} M_{\pi}^{2}
$$

where I have already plugged in the nonuniversal (i.e. $N_{f}$-dependent) constants for the 2-flavour case 18]. Relation (16) being the $\mathrm{QED}(2)$ analogue of the Gell-Mann-Oakes-Renner PCAC-relation

$$
m \Sigma \simeq \frac{1}{2} M_{\pi}^{2} F_{\pi}^{2} \quad\left(\forall N_{f}\right)
$$

in QCD means that I may define the LS-parameter in the lattice studies presented below as

$$
x \equiv \frac{0.388}{(2.008)^{2}} V M_{\pi}^{2} \quad\left(N_{f}=2\right)
$$

as one may rewrite it in the case of QCD (to leading order in XPT)

$$
x=\frac{1}{2} V M_{\pi}^{2} F_{\pi}^{2} \quad\left(\forall N_{f}\right)
$$

in a way which involves just physical degrees of freedom巴"

In order to investigate the LS-issue, I have chosen to implement the Schwinger model with a pair of (dynamical) staggered fermions, using the Wilson gauge action $S_{\text {gauge }}=\beta \sum\left(1-\cos \theta_{\square}\right)$. Since the staggered Dirac operator is represented by relatively small matrices (see below), I have decided to compute the determinants exactly, using the routines ZGEFA and ZGEDI from the LINPACK package.

I compare the three regimes $x \ll 1, x \simeq 1, x \gg 1$ to each other using three dedicated simulations: I have chosen to vary the box volume at fixed $\beta=1 /(a g)^{2}=3.4$ and fixed staggered quark mass $m=0.09$ (everything in lattice units). The three regimes are represented by the three volumes $V=8 \times 4, V=18 \times 6, V=40 \times 10$, where always periodic boundary conditions in the first (spatial) direction and thermal boundary conditions in the second (euclidean timelike) direction have been used. The LS-parameter (18) takes the values $x \simeq 0.33, x \simeq 1.12, x \simeq 4.16$ respectively, while the pion (pseudoscalar iso-triplet) has a (common) mass [2] $M_{\pi}=2.008 \cdot 0.542^{1 / 3} 0.09^{2 / 3}=0.329$ and hence a correlation length $\xi_{\pi}=3.04$ as to fit into the box (almost) in all

\footnotetext{
${ }^{11}$ Note that in order to determine $x$ in a lattice study through (19) one has to plug in $M_{\pi}$ and $F_{\pi}$ of the sea-pion, i.e. in practice of a pion constructed from current-quarks having exactly the same mass as their sea-partners.

${ }^{12}$ The formula used is the prediction (14), which seems adequate since $m / g \simeq 0.166 \ll 1$.
} 

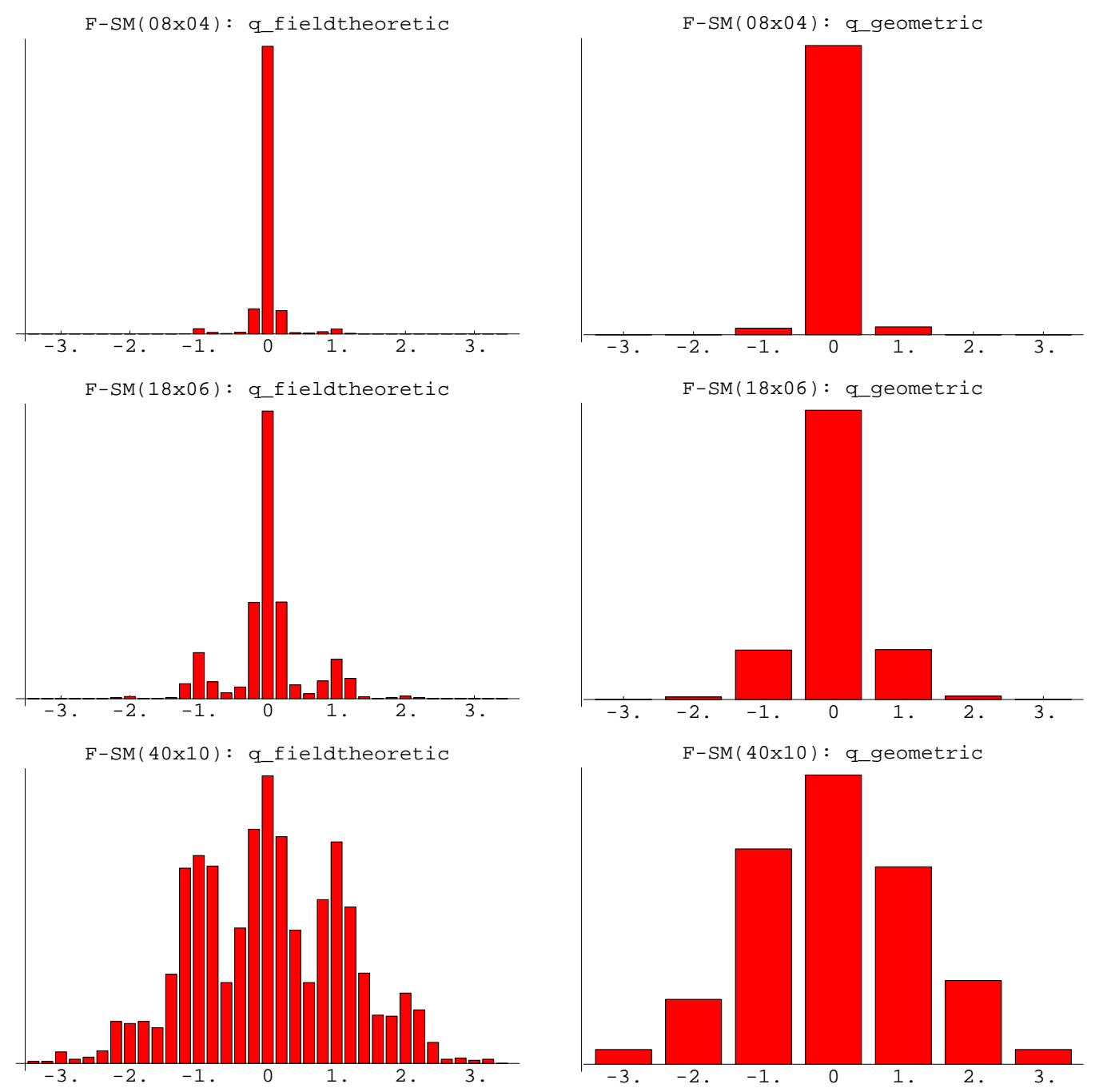

Figure 2: Distribution of $\nu_{\mathrm{fth}}$ (left) and $\nu_{\text {geo }}$ (right) in the small, intermediate and "large" Leutwyler-Smilga regimes, respectively.

three regimes - as is usual in a lattice context. Each time 3200 decorrelated (w.r.t. the plaquette) configurations have been generated.

For the type of investigation I am aiming at configurations must be assigned an index $\nu$. I have implemented both the geometric definition $\nu_{\text {geo }}=\frac{1}{2 \pi} \sum \log U_{\square}$ and the field theoretic definition $\nu_{\mathrm{fth}}=\kappa \nu_{\text {nai }}, \nu_{\text {nai }}=\sum \sin \theta_{\square}$ with the renormalization factor $\kappa \simeq 1 /\left(1-\left\langle S_{\text {gauge }}\right\rangle / \beta V\right)$ 19. A configuration is assigned an index only if the geometric and the field-theoretic definition, after rounding to the nearest integer, agree. This turned out to be the case for $3197,3163,2818$ configurations, i.e. on a $99.9 \%, 98.8 \%, 88.1 \%$ basis on the small/intermediate/large lattice. This fraction being so high means that in practice an assignment can be done without cooling for the overwhelming majority of configurations. The remaining ones are just not assigned an index; they are used to compute the unseparated observable (e.g. the "complete" heavy quark potential; see below), the latter, however, turns out to be unaffected by whether they are included or not.

Having simulation data which are supposed to reflect the situation in the 
small/intermediate/large LS-regimes respectively, one may want to check the overall distribution of topological charges as encountered in the three runs. As one can see from the r.h.s. of Fig. 2, the overall distribution is noticeably different in the three regimes. The predictions by Leutwyler and Smilga for the two extreme cases $(x \ll 1$ and $x \gg 1)$ seem to be well reproduced: For small $x$ the partition function $Z$ (and hence the sample) is dominated by $Z_{0}$, the contribution from the topologically trivial sector, whereas for large $x$ the distribution gets broad and seems compatible with a gaussian. It is worth mentioning that the latter holds true even though the pion did not overlap the box; rather the usual relation $\xi_{\pi} \ll L$ applied. This is a first indication that the r.h.s. of (8) in the LS-analysis might indeed be a purely technical condition, immaterial to what they claim, namely that in the large $x$ regime the topological charge is "an irrelevant concept" or, in other words, that physical observables do not depend on $\nu$ if $x \gg 1$ [6].

\section{Sectoral reweightings and renormalizations}

Since the LS-issue is peculiar to the full (unquenched) theory, an attempt to understand by which mechanism the three regimes differ from each other may lead one to investigate how the functional determinant or specifically its contribution to the total action per continuum-flavour

$$
S_{\text {fermion }}=-\log (\operatorname{det}(\not D+m))+\text { const }
$$

relates to the contribution from the gauge field and, in addition, how such a relationship might depend on the topological charge of the background. The idea is thus to study such a relationship sectorally, i.e. after the complete sample has been separated into subsamples with a fixed topological charge. Since the theory (at $\theta=0$ ) is $\mathrm{P} / \mathrm{CP}$-symmetric, the configurations from the sectors $\pm \nu$ may be combined into a single subsample characterized by $|\nu|$.

As one can see from the rightmost column of Fig. 3, the contribution per continuum flavour to the total action, $S_{\text {fermion, }}$, shows a positive correlation with the gauge action $S_{\text {gauge }}$. This means that, on a qualitative level, switching on the functional determinant amounts to an increase of $\beta$, which is a well-known feature in (full) QCD too. However, the correlation is weak, i.e. the "cigars" in the unseparated plots are thick. A key observation is that the correlation improves, if one separates the sample into subsamples with fixed $|\nu|$, as is done in Fig. 3. It is worth noticing that in general (i.e. without specifying the LS-regime) the best linear fit for $S_{\text {fermion }}$ as a function of $S_{\text {gauge }}$ depends (in offset and slope) on $|\nu|$. This means that, to a first approximation, the functional determinant results in an overall suppression of higher topological sectors w.r.t lower ones and to a sectorally different renormalization of $\beta$. As we shall see, these two features constitute the place where the three LS-regimes differ from each other.

First, we shall consider the offset of the regression line in the $\nu= \pm 1$ sectors compared to the line in the sector with $\nu=0$, as this is a measure how 


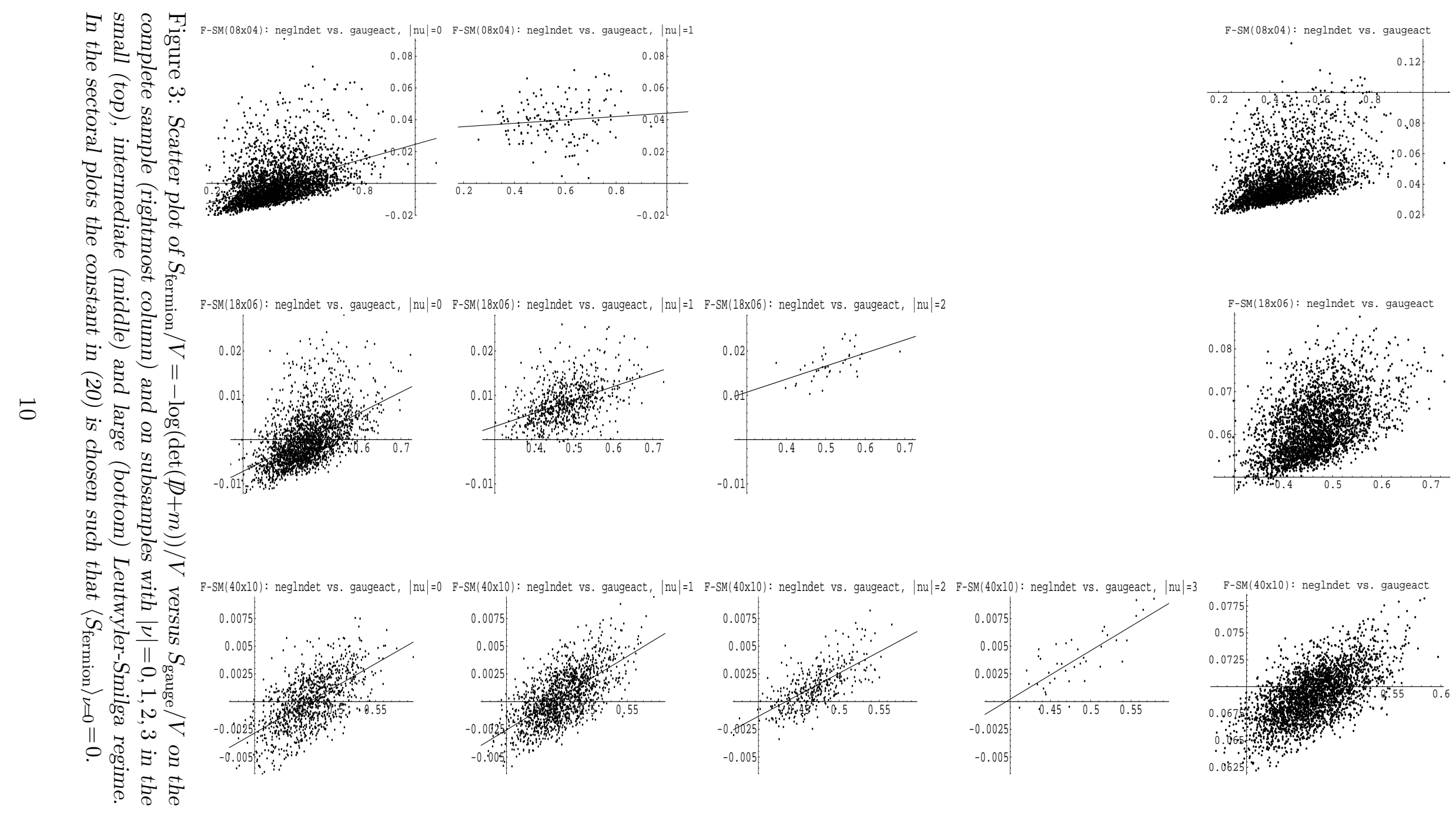


much extra suppression the first topologically nontrivial sector receives on average compared to the trivial one due to the functional determinant. From the first and second column in Fig. 3 one sees that this offset (and in general the offset between neighboring sectors) decreases with increasing $x$. This provides an explanation [20] for the broadening of the $\nu$-distribution inherent to passing from the small to the intermediate or from the intermediate to the large LS-regime (cf. Fig. 2). For $x \ll 1$ the offset $\left\langle S_{\text {fermion }}\right\rangle_{1}-\left\langle S_{\text {fermion }}\right\rangle_{0}$ is larger than the fluctuations in $S_{\text {fermion }}$ within the zero-charge sector, and this means means that in the small LS-regime the functional determinant acts as an almost strict constraint to the topologically trivial sector. For $x \simeq 1$ the offset has roughly the same size as typical fluctuations of $S_{\text {fermion }}$ in the sector with $\nu=0$; as a result of this one can say that in the intermediate regime the functional determinant acts as a "soft constraint" to low-lying $|\nu|$ values. For $x \gg 1$ finally, $\left\langle S_{\text {fermion }}\right\rangle_{1}-\left\langle S_{\text {fermion }}\right\rangle_{0}$ is small compared to the fluctuations in each one of these sectors; hence no such effective description proves appropriate in the large LS-regime.

Passing on to the slope of the regression line in the correlation $\left\langle S_{\text {fermion }}\right\rangle$ versus $\left\langle S_{\text {gauge }}\right\rangle$, we notice from Fig. 3 that this slope decreases (in general) with increasing $|\nu|$. This means that the effective renormalization

$$
\beta^{(\nu)} \longrightarrow \beta_{\mathrm{eff}}^{(\nu)}=\left(1+N_{f} \operatorname{slope}(\nu)\right) \beta^{(\nu)}
$$

due to the functional determinant is (in general) stronger for sectors with low absolute value of the net topological charge. This effect of sectoral dependence of the effective renormalization factor for $\beta$ diminishes with increasing $x$; eventually, in the large $x$ regime, it is completely gone.

Thus, the large LS-regime is unique, as the determinant results only in an overall suppression of higher topological sectors, but not in a sectoral dependence of $\beta_{\text {eff }}$ (and hence, in 4 dimensions, of the lattice spacing in physical units). This seems consistent with the claim by Leutwyler and Smilga that physical observables do not depend on $\nu$ in the large $x$ regime, but it is of course not a sufficient condition for the latter to be true.

\section{$5 \quad$ Sectoral heavy quark free energies}

A quantity which is easily accessible in lattice studies and which might help to elucidate the physical meaning of the LS-classification is the Polyakov loop, i.e. the trace of a chain of link variables which winds once around the torus in the euclidean timelike direction. Its logarithm represents (up to a factor) the free energy of an external ("heavy") quark which is brought into the system without possibility to influence it through back-reaction. As in the previous section the analysis shall be done sectorally, i.e. on classes of configurations with a fixed value of $|\nu|$.

The result of such an analysis for the Polyakov loop is displayed in Fig. 4. As one can see from the rightmost column, the expectation value $\langle L\rangle$ on the "complete" (unseparated) sample decreases if the box volume is increased. 


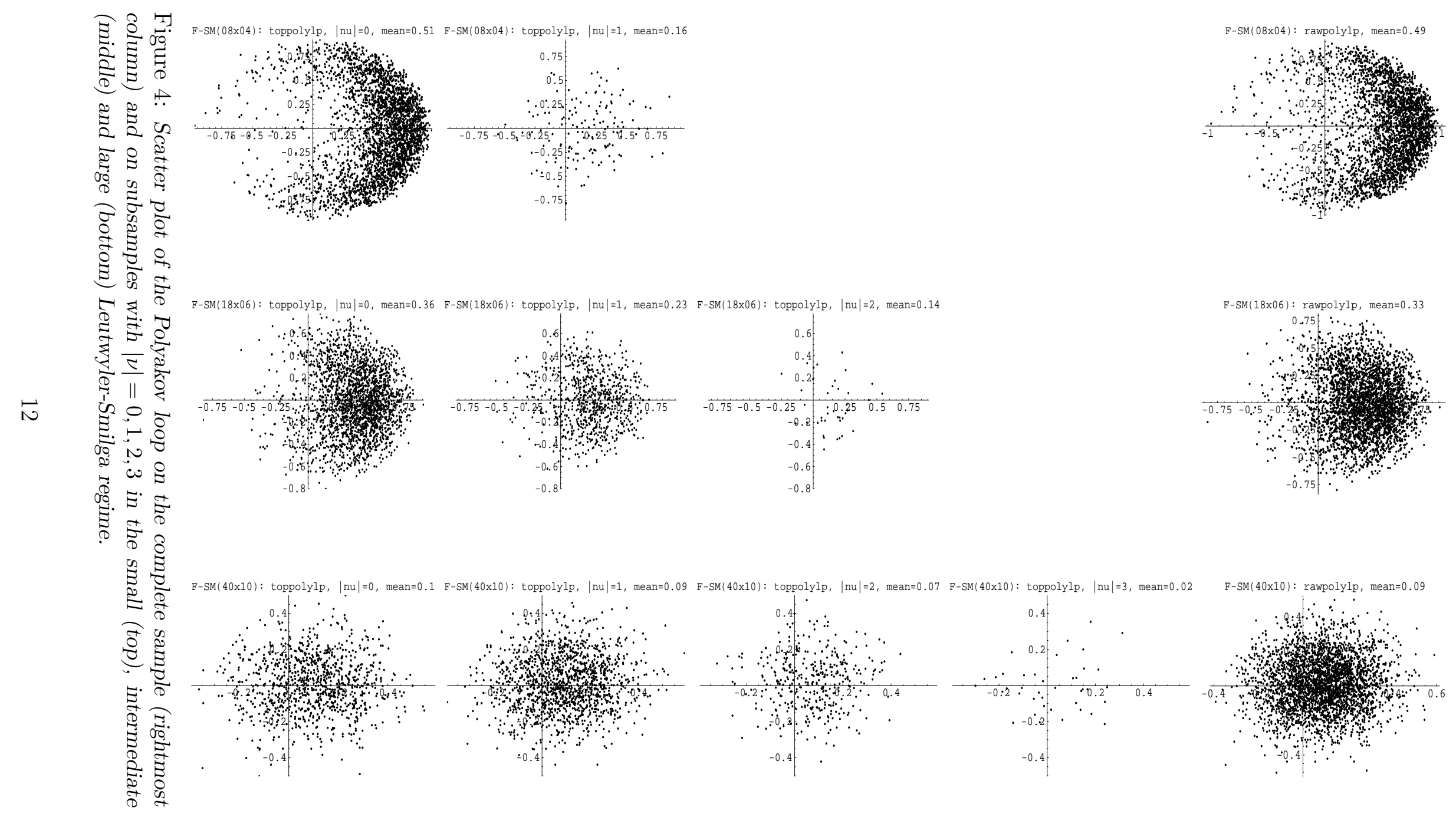


What is more interesting is the following: The physical (unseparated) expectation value may be understood as a weighted mean

$$
\langle L\rangle=\sum_{|\nu| \geq 0} p_{|\nu|}\langle L\rangle_{|\nu|}
$$

of sectoral expectation values $\langle L\rangle_{|\nu|}$, where the factor $p_{|\nu|}$ reflects the combined weight of the sectors $\pm \nu$ in the histogram on the r.h.s. of Fig. 2. From the first line in Fig. 3 one notices that the sectoral expectation values for $|\nu|=0,1$ in the small LS-regime differ quite drastically on the scale set by the physical expectation value, i.e. $\left|\langle L\rangle_{|0|, x \ll 1}-\langle L\rangle_{|1|, x \ll 1}\right| /\langle L\rangle_{x \ll 1} \simeq 0.7$. In the intermediate regime the analogous ratios for neighboring sectors (i.e. $\left|\langle L\rangle_{|0|, x \simeq 1}-\langle L\rangle_{|1|, x \simeq 1}\right| /\langle L\rangle_{x \simeq 1}$ and $\left.\left|\langle L\rangle_{|1|, x \simeq 1}-\langle L\rangle_{|2|, x \simeq 1}\right| /\langle L\rangle_{x \simeq 1}\right)$ happen to be smaller, but comparing the highest to the lowest available $|\nu|$, one finds again $\left|\langle L\rangle_{|0|, x \simeq 1}-\langle L\rangle_{|2|, x \simeq 1}\right| /\langle L\rangle_{x \simeq 1} \simeq 0.7$. Finally, in the large LS-regime, there is an almost perfect consistency between sectoral Polyakov loop expectation values for low-lying $|\nu|$ (i.e. $|\nu| \in\{0,1,2\}$ ), but there is no numerical evidence in favour of an overall consistency; if one builds the analogous ratio for the lowest and highest $|\nu|$ available, the quantity $\left|\langle L\rangle_{|0|, x \gg 1}-\langle L\rangle_{|3|, x \gg 1}\right| /\langle L\rangle_{x \gg 1}$ is found to be again of order one.

The Polyakov loop is the first example of a physical observable for which we see a difference between expectation values in neighboring sectors (for sufficiently low $|\nu|$ values) disappear upon taking the large $x$ limit. However, even in the large LS-regime no supporting evidence has been found that $\langle L\rangle_{\nu}$ might be completely independent of $\nu$. It seems that $\langle L\rangle_{|\nu|}$ proves sensitive on topology even in the large LS-regime when jumping from $|\nu|_{\min }$ to $|\nu|_{\max }$.

\section{Sectoral heavy quark potentials}

The second observable which has been evaluated for each sector separately is the heavy quark potential as determined from the correlator of two Polyakov loops - see Fig. 5 and Tabs. 1-3. The first thing to notice is that in each regime the physical potential (the one in the rightmost column in Fig. 5) bends to the right. This curvature is a direct indication of the screening brought by the dynamical fermions; the quenched potential is strictly linear ${ }^{133}$.

In the small LS-regime the sectoral potentials for $\nu=0$ and $\nu= \pm 1$ differ quite drastically. In this case the potential in the topologically trivial sector agrees (on a $1 \sigma$ basis, cf. Tab. 1) with the potential on the full sample - as expected, since for $x \ll 1$ the partition function is dominated by $Z_{0}$. What might come as a surprise is that the error-bars in the first plot in the top line of Fig. 5 are smaller than those in the rightmost one, even though the configurations analyzed in the former case represent a subset of those used in the latter case. The reason, as we have seen, is that the complementary subset (the one comprising the configurations with $\nu= \pm 1$ ) provides measurement

\footnotetext{
${ }^{13}$ Unlike in $\mathrm{QCD}(4)$ there is no $1 / r$ short-distance tail; the one-photon exchange contributes a linear piece in 2 dimensions.
} 


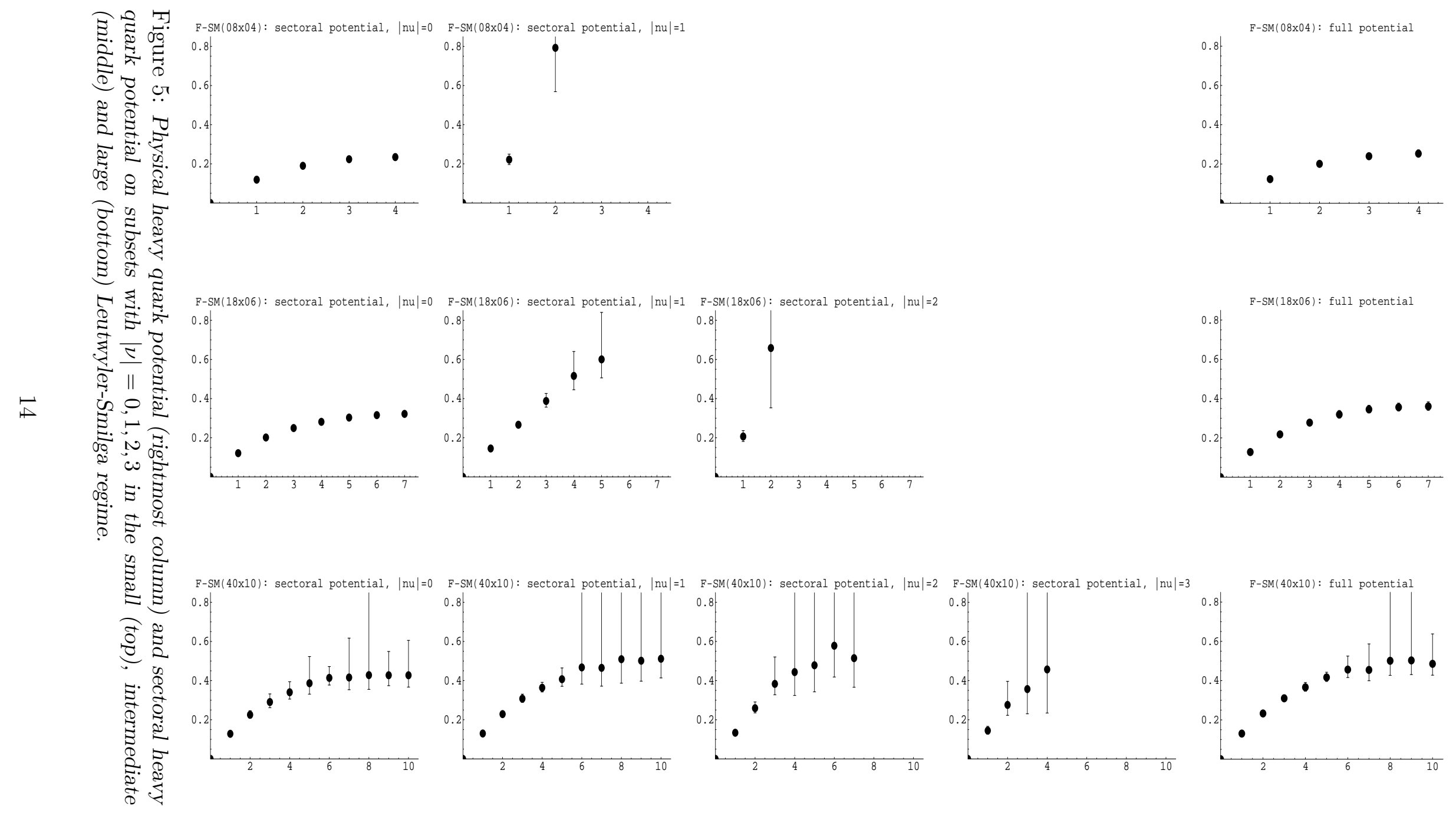




\begin{tabular}{|c|ccc|}
\hline & dist $=1$ & dist $=2$ & dist $=3$ \\
\hline$|\nu|=0$ & $0.119_{-0.003}^{+0.003}$ & $0.190_{-0.009}^{+0.009}$ & $0.224_{-0.012}^{+0.013}$ \\
$|\nu|=1$ & $0.222_{-0.025}^{+0.028}$ & $0.793_{-0.224}^{+\infty}$ & indet. \\
\hline all conf. & $0.123_{-0.003}^{+0.003}$ & $0.201_{-0.009}^{+0.010}$ & $0.240_{-0.015}^{+0.016}$ \\
\hline
\end{tabular}

Table 1: Sectoral heavy quark potentials and unseparated (physical) potential at spatial separations $1 . .3$ in the small Leutwyler Smilga regime $(x \ll 1)$.

\begin{tabular}{|c|cccc|}
\hline & dist $=1$ & dist $=2$ & dist $=3$ & dist $=4$ \\
\hline$|\nu|=0$ & $0.121_{-0.002}^{+0.002}$ & $0.201_{-0.004}^{+0.004}$ & $0.250_{-0.006}^{+0.006}$ & $0.281_{-0.011}^{+0.012}$ \\
$|\nu|=1$ & $0.145_{-0.005}^{+0.005}$ & $0.266_{-0.015}^{+0.015}$ & $0.388_{-0.031}^{+0.038}$ & $0.516_{-0.071}^{+0.125}$ \\
$|\nu|=2$ & $0.206_{-0.025}^{+0.030}$ & $0.658_{-0.305}^{+\infty}$ & indet. & indet. \\
\hline all conf. & $0.128_{-0.002}^{+0.002}$ & $0.218_{-0.007}^{+0.008}$ & $0.278_{-0.012}^{+0.013}$ & $0.319_{-0.018}^{+0.020}$ \\
\hline
\end{tabular}

Table 2: Sectoral heavy quark potentials and unseparated (physical) potential at separations $1 . .4$ in the intermediate Leutwyler Smilga regime $(x \simeq 1)$.

\begin{tabular}{|c|ccccc|}
\hline & dist $=1$ & dist $=2$ & dist $=3$ & dist $=4$ & dist $=5$ \\
\hline$|\nu|=0$ & $0.128_{-0.006}^{+0.006}$ & $0.226_{-0.017}^{+0.021}$ & $0.291_{-0.029}^{+0.041}$ & $0.340_{-0.035}^{+0.054}$ & $0.387_{-0.056}^{+0.136}$ \\
$|\nu|=1$ & $0.130_{-0.003}^{+0.004}$ & $0.229_{-0.007}^{+0.008}$ & $0.308_{-0.019}^{+0.023}$ & $0.364_{-0.022}^{+0.028}$ & $0.407_{-0.036}^{+0.057}$ \\
$|\nu|=2$ & $0.134_{-0.006}^{+0.007}$ & $0.259_{-0.024}^{+0.032}$ & $0.383_{-0.056}^{+0.137}$ & $0.444_{-0.119}^{+\infty}$ & $0.478_{-0.136}^{+\infty}$ \\
$|\nu|=3$ & $0.145_{-0.018}^{+0.022}$ & $0.276_{-0.053}^{+0.120}$ & $0.357_{-0.126}^{+\infty}$ & $0.457_{-0.223}^{+\infty}$ & indet. \\
\hline all conf. & $0.130_{-0.003}^{+0.003}$ & $0.233_{-0.010}^{+0.011}$ & $0.310_{-0.015}^{+0.018}$ & $0.366_{-0.020}^{+0.025}$ & $0.417_{-0.021}^{+0.026}$ \\
\hline
\end{tabular}

Table 3: Sectoral heavy quark potentials and unseparated (physical) potential at spatial separations $1 . .5$ in the large Leutwyler Smilga regime $(x \gg 1)$.

data which are inconsistent with the data won from the topologically trivial subset, but has a total weight which is too small to affect the complete ensemble average significantly (cf. the first plot in Fig. 2). Hence, in the small LS-regime, the tiny contribution from the higher topological sectors primarily adds some noise to a standard observable like the heavy quark potential. The practical recipe to get rid of this effect is to do by hand (for sufficiently small $x$ ) what the functional determinant does anyways in the limit $x \rightarrow 0$ : Cut away the higher topological sectors !

In the intermediate LS-regime the difference between the two sectoral potentials for $\nu=0$ and $\nu= \pm 1$ is smaller than in the previous regime, but the sectoral potentials are typically several $\sigma$ away from each other and even the potential in the topologically trivial sector lies significantly below the unseparated physical potential (cf. Tab. 2). From this we see that the sector with $\nu=0$ does not always yield the right result. The intermediate LS-regime shares thus with the small $x$ regime the uneasy property that different sectors give inconsistent values for physical observables, but unlike in the small $x$ regime, several sectors yield sizable contributions to the complete (physical) expectation value - hence perfect ergodicity w.r.t. $\nu$ is mandatory. 
In the large LS-regime differences between expectation values measured on subsets of definite topological charge diminish further, if the latter differs only by one ("neighboring sectors"). On the other hand, in the large $x$ regime there is a multitude of topological sectors yielding sizable contributions to the physical expectation value, hence an obvious question is which effect will win in the limit $x \rightarrow \infty$. Hence, what we would like to know is whether the difference between the heavy quark potential on the subsets with the lowest and the highest (reasonably populated) $|\nu|$ will fade away, stay constant or grow if $x$ increases unlimitedly. From Tab. 3, one gets the impression that the difference between the two extreme fixed- $|\nu|$ expectation values persists in the large LS-regime. It seems that the statement by Leutwyler and Smilga that in the large $x$ regime the topological charge has vanishing influence on physical measurements is reproduced for sufficiently low $|\nu|$ values (in our case e.g. $|\nu| \in\{0,1,2\}$ but not necessarily for $|\nu|=3$ ). It is also worth noticing that the topologically trivial potential lies at least $0.5 \sigma$ below the physical potential. Hence, a constraint to the topologically trivial sector seems permissible for $x \gg 1$, though in our simulation a constraint to the sectors $|\nu|=1$ would have been a better choice. Of course it is difficult to get definitive answers from numerical data; it may well be that our LS-value $(x \simeq 4.16)$ is still too small for the large $x$ behaviour to be fully pronounced. Nevertheless, the situation seems to be analogous to what we have seen in the case of the heavy quark free energy: Any differences between expectation values of neighboring sectors (with sufficiently low $|\nu|$ ) disappear upon taking the large $x$ limit. However, even in the large LS-regime no supporting evidence has been found that $\left\langle V_{q \bar{q}}\right\rangle_{\nu}$ might be completely independent of $\nu$.

\section{Summary}

The massive multi-flavour Schwinger model has been used to test the statements by Leutwyler and Smilga about the (ir-)relevance of the topological charge for QCD in a finite box. It is expected that an analogous study for full QCD will result in figures which look, as far as qualitative issues go, exactly the same as the ones presented here and that, for this reason, the statements below are valid for QCD too. The key points are the following:

(i) The three LS-regimes show distinctive properties already on a formal level, by the way how the contribution per flavour to the total action (the sign-flipped logarithm of the one-flavour functional determinant) correlates with the contribution from the gauge field. Introducing the fermion determinant at fixed $\beta$ results in every regime in an overall suppression of higher topological sectors w.r.t. low-lying ones. For $x \ll 1$ this suppression is so strong that the functional determinant effectively acts as a constraint to the topologically trivial sector. In addition, the sea-flavours effectively result in a sectoral multiplicative renormalization of $\beta$ with a factor greater than 1 . For small and intermediate $x$ this factor is found to decrease as a function of $|\nu|$, while in the large LS-regime the renormalization factor seems to be uniform for all topological sectors. 
(ii) For the overall distribution of topological charges the predictions by Leutwyler and Smilga are well observed: In the regime $x \ll 1$ the partition function (and hence the sample) is entirely dominated by the topologically trivial sector, i.e. the histogram of topological charges essentially consists of a delta-peak at $\nu=0$. In the intermediate regime $(x \simeq 1)$ the distribution is narrow but nontrivial, i.e. sizable contributions stem essentially from the sectors $\nu=0, \pm 1, \pm 2$. In the regime $x \gg 1$ the topological charge distribution gets broad and is approximated by a gaussian with width $\left\langle\nu^{2}\right\rangle \simeq x / N_{f}$.

(iii) In the small LS-regime $(x \ll 1)$ the sectoral expectation value of an observable in the first topologically nontrivial sector (typically won from very few configurations) differs drastically from the analogous expectation value in the topologically trivial sector (represented by the overwhelming majority of configurations). This means that a simulation getting stuck at $\nu= \pm 1$ (or even higher) is absolutely disastrous to the result, i.e. tiny non-ergodicities w.r.t. the topological charge render an overall sample average meaningless. The good news is that in this regime the correct distribution is known and hence there is a simple remedy: A strict constraint to the topologically trivial sector proves beneficial.

(iv) The intermediate LS-regime $(x \simeq 1)$ is characterized by the distinctive feature that several mutually inconsistent sectors yield sizable contributions to a standard (i.e. not relating to the $U(1)_{A}$ issue) physical observable. This means that the intermediate regime is the one which is most delicate to simulate in: There is a strong sectoral dependence of physical observables, yet there is no simple recipe how to guarantee the correct sectoral weighting. In other words: Physical measurements on a sample won in the intermediate LSregime do rely on the simulation algorithm having achieved perfect ergodicity w.r.t. the topological charge.

$(v)$ In the large LS-regime $(x \gg 1)$ sectoral averages seem consistent between neighboring topological sectors (for sufficiently low $|\nu|$ ), but no evidence has been found that measurements might be completely independent of $\nu$. It seems that jumping (within a well-distributed sample) from the lowest to the highest accessible $|\nu|$ may affect sectoral averages even in the large $x$ regime. This, if correct, means that the statement by Leutwyler and Smilga according to which "the topological charge is an irrelevant concept in the large $x$ regime" [6] should to be interpreted in the following way: In the large LSregime physical observables are immune to modifications of the $\nu$-histogram which are small compared to its natural width $\left(x / N_{f}\right)^{1 / 2}$; they may, however, be sensitive to distortions which go beyond this limit. From a lattice perspective the implication is that the result of a full QCD simulation in the large $x$ regime may be trusted without hesitation as long as the algorithm did not get stuck in a sector with unreasonably high $|\nu|$, say at $\nu=10$ if the simulation was in the regime $x \sim 40$ and $N_{f}=2$.

(vi) In this work, the transition from the small to the intermediate and the large LS-regime has been made by adding more sites to the grid while keeping $\beta, m$ fixed. It would be interesting to test the universality property, i.e. the claim that it is only the product $V \Sigma m[6]$ which decides on the regime. 
(vii) It is worth emphasizing that in the large $x$ simulation used in this work the predictions by Leutwyler and Smilga turned out to be fulfilled even though the condition in the r.h.s. of (8) according to which the pion would overlap the box was not obeyed, but rather the opposite relation $1 / M_{\pi} \ll L$ would hold true, i.e. the pion would fit into the box, as is usual in a lattice context. Hence the r.h.s. of (8) seems to be a purely technical condition, immaterial to the result of the LS-analysis in the large $x$ regime.

(viii) From the fact that the massive multi-flavour Schwinger model follows the LS-classification even though it resembles QCD slightly above the chiral phase transition one is led to suspect that this classification might be more general, even within QCD, than its original derivation indicates. In other words: The conjecture is that the LS-classification may prove useful not only in the broken phase but also in the high-temperature phase of QCD as long as one stays in the immediate vicinity of the phase transition, i.e. as long as pions keep being visible (i.e. keep dominating the long-range Green's functions with pion-type quantum numbers; for a recent study see e.g. [21]). This, if correct, would imply that the version of the LS-parameter as given in eqn. (19) is more general ${ }^{4}$ then the original version (2).

(ix) The results of this investigation may be seen as an additional aposteriori justification of the resources phenomenological lattice groups have been provided with in order to be able to study (full) QCD in the large $x$ regime可. In addition, they support attempts to develop algorithms which decorrelate the topological charge faster than standard HMC (see e.g. [22]), as this is necessary for trustworthy simulations in the intermediate LS-regime, towards which one moves when comparing different runs with increasing $\kappa_{\text {sea }}$ in a fixed physical volume, or even more so, if $\beta$ is kept fixed. The fact that even in the large $x$ regime full QCD simulations may yield unreliable results upon generating extreme distortions in the topological charge distribution should be taken as a motivation to monitor $\nu$ in all phenomenological studies (i.e. not just with an observable relating to the $U(1)_{A}$ issue) and, for the time being, as a warning against pushing the sea-quark mass too light ${ }^{10}$ (even when increasing the volume together with $\kappa_{\text {sea }}$ so as to keep the product $x=V \Sigma m$ fixed and large compared to 1). The latter constraint is certainly not a disaster, since there is a well-elaborate framework [23] which allows one to extrapolate from samples generated with a somewhat larger sea-quark mass down to the physical $M_{\pi} / M_{\rho}$. Hence it seems that our results do represent a little caveat but no real obstacle to future progress in Lattice QCD.

\section{Acknowledgements}

I would like to thank Steve Sharpe for fruitful physics discussions in an early phase of this work and Roland Rosenfelder for advice regarding presentation.

\footnotetext{
${ }^{14}$ Note that in the high-temperature phase $\Sigma=0$, hence (2) is useless for $T>T_{c}$.

${ }^{15}$ This is the result of a private attempt to estimate $x$ in dynamical simulations aiming at the light hadron spectrum from published data (e.g. $m_{\text {sea }}$ and $a$ in physical units).

${ }^{16}$ Here it is assumed that each algorithm has a critical $M_{\pi} / M_{\rho}$ (which may well depend on the actions used) beneath which it tends to get stuck (cf. footnote 1).
} 


\section{References}

[1] T. Schäfer and E.V. Shuryak, Rev. Mod. Phys. 70, 323 (1998).

[2] Y. Kuramashi et al., Phys. Lett. B 313, 425 (1993); M. MuellerPreussker, Proc. of XXVI Int. Conf. on High Energy Physics, Dallas, TX, 1992, ed. J.R. Sanford, AIP Conf. Proc. No. 272, 1545 (1993); B. Allés et al., Phys. Lett. B 389, 107 (1996).

[3] B. Allés et al., Phys. Rev. D 58, 071503 (1998).

[4] B. Allés et al., Proc. of ICHEP 96 Warsaw, 1599 (1996).

[5] M. Fukugita et al., Phys. Rev. D 51, 3952 (1995); A. Ali Khan et al. (CP-PACS), Nucl. Phys. (Proc. Suppl.) 83, 162 (2000).

[6] H. Leutwyler and A.V. Smilga, Phys. Rev. D 46, 5607 (1992).

[7] P.H. Damgaard, Nucl. Phys. B 556, 327 (1999).

[8] C. Vafa and E. Witten, Nucl. Phys. B 234, 173 (1984).

[9] T. Banks and A. Casher, Nucl. Phys. B 169, 103 (1980).

[10] S. Descotes and J. Stern, hep-ph/9912234.

[11] J. Gasser and H. Leutwyler, Phys. Lett. B 188, 477 (1987).

[12] H. Joos, Helv. Phys. Acta 63, 670 (1990); I. Sachs and A. Wipf, Helv. Phys. Acta 65, 652 (1992).

[13] S. Coleman, Comm. Math. Phys. 31, 259 (1973).

[14] A.V. Smilga and J.J.M. Verbaarschot, Phys. Rev. D 54, 1087 (1996).

[15] G. Segrè and W.I. Weisberger, Phys. Rev. D 10, 1767 (1974).

[16] A.V. Smilga, Phys. Lett. B 278, 371 (1992).

[17] M.A. Shifman and A.V. Smilga, Phys. Rev. D 56, 7978 (1997); S. Dürr, Ann. Phys. 273, 1 (1999).

[18] A.V. Smilga, Phys. Rev. D 55, 443 (1997).

[19] M. Lüscher, Comm. Math. Phys. 85, 39 (1982); J. Smit and J.C. Vink, Nucl. Phys. B 303, 36 (1988); J.C. Vink, Nucl. Phys. B 307, 549 (1988).

[20] C.R. Gattringer, I. Hip and C.B. Lang, Phys. Lett. B 409, 371 (1997), Nucl. Phys. B 508, 329 (1997); C.R. Gattringer and I. Hip, Nucl. Phys. B 536, 363 (1998).

[21] Ph. de Forcrand et al., hep-lat/0008005.

[22] E.M. Ilgenfritz et al., hep-lat/0007039.

[23] S. Sharpe and N. Shoresh, hep-lat/0006017. 